\title{
Classical and Non-Classical Effective Medium Theories: New Perspectives
}

\author{
Igor Tsukerman \\ Department of Electrical and Computer Engineering, The University of Akron \\ Akron,OH 44325-3904,USA. igor@uakron.edu
}

\begin{abstract}
Future research in electrodynamics of periodic electromagnetic composites (metamaterials) can be expected to produce sophisticated homogenization theories valid for any composition and size of the lattice cell. The paper outlines a promising path in that direction, leading to non-asymptotic and nonlocal homogenization models, and highlights aspects of homogenization that are often overlooked: the finite size of the sample and the role of interface boundaries. Classical theories (e.g. Clausius-Mossotti, Maxwell Garnett), while originally derived from a very different set of ideas, fit well into the proposed framework. Nonlocal effects can be included in the model, making an order-of-magnitude accuracy improvements possible. One future challenge is to determine what effective parameters can or cannot be obtained for a given set of constituents of a metamaterial lattice cell, thereby delineating the possible from the impossible in metamaterial design.
\end{abstract}

Keywords: Maxwell's equations, metamaterials, homogenization, effective medium theory, boundary conditions

\section{Introduction}

Electromagnetic metamaterials are artificial periodic structures engineered to control the propagation of waves and to achieve physical effects not attainable in natural materials - high-frequency magnetism, negative refraction, strong absorption, lensing, cloaking, and more. Research in metamaterials started three decades ago [1], if not earlier, and exploded in the 2000s as a quest for "perfect lenses," "perfect absorbers," etc. But, as the field of metamaterials matured, it became clear that ideal devices were not realizable because of losses, finite lattice cell sizes [2], and other factors. Undoubtedly, however, "imperfect" materials and devices will continue to be developed, and we can therefore expect a growing need for more sophisticated methods of their analysis and, more specifically, for accurate homogenization theories valid for any composition and size of the lattice cell. The objective of homogenization (effective medium theory) is to describe a composite structure in terms of effective parameters accurately representing reflection, transmission and propagation of waves on the scale coarser than the lattice cell size.

Most mathematical theories of homogenization are asymptotic - i.e. valid in the limit of the lattice cell size vanishingly small relative to some characteristic scale. (For wave problems, this scale is the free-space wavelength; in statics, it is the scale of variation of the applied field and/or the size of the material sample.) It is now understood, however, that in this asymptotic limit all nontrivial properties of metamaterials - including, notably, magnetic response and negative refraction - vanish $[2,3,4]$. Hence a non-asymptotic treatment is called for.

Effective medium theories of classical physics (Clausius-Mossotti, Lorenz-Lorentz, Maxwell Garnett) rely on simplification assumptions that work very well for relatively simple mixtures but require extensions and enhancements $[5,6,7]$ in more complicated cases. 
As argued in the present paper, a promising and inexorable direction of future research is non-asymptotic and possibly nonlocal homogenization. The paper has three main parts. The first one is a condensed review of the homogenization framework of [8]. The second part extends the non-asymptotic framework to a nonlocal one. Finally, we explore the connection between non-asymptotic homogenization and classical theories of the $19^{\text {th }}-$ early $20^{\text {th }}$ century. While our perspective is very different from that of $19^{\text {th }}$ century physics, we shall see that classical theories fit in nicely with the new framework. Perspectives for future research are outlined in the Conclusion.

Informally, the essence of the problem is illustrated by Fig. 1. A given periodic structure is to be replaced with a homogeneous sample of the same geometric shape and size, with some material tensor $\mathcal{M}$ to be defined, in such a way that reflection and transmission of waves (or, equivalently, the far-field pattern) remain, to the extent possible, unchanged. This informal description is made more precise in [8].

Homogenization is fundamentally a nonlinear inverse problem which in each particular case could in principle be solved by brute numerical force. However, this is very expensive computationally (especially for large samples) and - perhaps more importantly - gives little insight into the physical behavior of waves in the material and into its design. Instead, we solve this inverse problem approximately, using, as

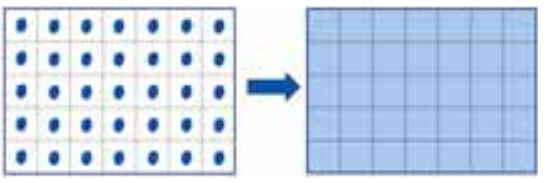

Figure 1. A periodic structure replaced with an equivalent (in the sense explained in the text) homogeneous sample. Coarse-level fields should be defined in such a way as to satisfy Maxwell's equations and interface boundary conditions exactly or as accurately as possible.

\section{Key Ideas of Non-asymptotic Homogenization}

We shall use the tilde sign to indicate periodic functions over orthorhombic lattices:

$$
\tilde{f}\left(x+n_{x} a_{x}, y+n_{y} a_{y}, z+n_{z} a_{z}\right)=\tilde{f}(x, y, z),
$$

where $a_{x, y, z}$ are the lattice periods and $n_{x, y, z}$ are arbitrary integers. (All such periodic functions are restricted to the domain occupied by the material.) The intrinsic permittivity $\tilde{\epsilon}(\mathbf{r})$ is assumed to be lattice-periodic and scalar, while the intrinsic magnetic permeability of all material constituents is unity, $\tilde{\mu}(\mathbf{r})=1$. Let the sample be illuminated by monochromatic waves with a given far-field pattern; these waves are reflected by the metamaterial. Our time-phasor convention is $\exp (-i \omega t)$.

The problem has two principal scales (levels). Fine-level fields are the exact solutions of Maxwell's equations for given illumination conditions and for a given sample. These fields are denoted with small letters $\mathbf{e}, \mathbf{d}, \mathbf{h}$ and $\mathbf{b}$. In general, their variation in space is rapid and consistent with the microstructure of metamaterial cells. Coarse-level fields vary on a characteristic scale greater that the cell size. Importantly, there are two distinct types of such fields:

(i) The actual physical fields in and around a (hypothetical) homogeneous sample. These can be obtained (e.g. numerically) only after the equivalent material tensor has been determined, and therefore cannot play a role in any valid homogenization procedure free of circular reasoning.

(ii) Some smoothed (averaged) versions of the fine-level ones. In contrast with (i), such fields are auxiliary mathematical constructions rather than measurable physical quantities. ${ }^{1}$ These auxiliary fields are denoted with capital letters $\mathbf{E}, \mathbf{D}, \mathbf{H}, \mathbf{B}$ and depend on the respective fine-scale fields. Although the latter are not known a priori, suitable approximations can be used in their stead, as explained below.

The key requirement for constructing coarse-level fields is that they satisfy Maxwell's equations and all interface boundary conditions as accurately as possible. Importantly, effective magnetic properties of metamaterials cannot be determined from the bulk behavior alone as a matter of principle. This is due to the fact that the Maxwell equation

\footnotetext{
${ }^{1}$ To quote [9, pp. 42-43]: "We now indicate why fields inside materials are impossible to measure.... within the polarized body the [coarselevel] fields are mathematical constructs defined in terms of equivalent smoothed charge and current distributions. A measurement of an electric or magnetic field inside the ... body would not necessarily measure these fields because the spatial and time averages taken by a probe, such as an energetic electron beam shot through the body or electrons of a conduction current, are not necessarily the same as the averages implied in [macroscopic Maxwell's equations]. ... the fields predicted from [macroscopic Maxwell's equations] have physical meaning only in the space surrounding a polarized body or in slots cut into the body ... but have no direct physical meaning inside the body itself."
} 
$\nabla \times \mathbf{H}=-i k_{0} \mathbf{D}$ is invariant with respect to an arbitrary simultaneous rescaling of vectors $\mathbf{H}$ and $\mathbf{D}$. (Equivalently, the splitting of the total current into "polarization" and "magnetization" components is not unique.) Loosely speaking, bulk behavior defines the dispersion relation only, while magnetic characteristics depend on the boundary impedance as well.

Unless the metamaterial is homogeneous to begin with, some approximation errors are unavoidable and need to be minimized. A physical interpretation of the source of these errors is spurious electric and magnetic currents [8]

$$
\delta \mathbf{J}_{m}(\mathbf{r})=\nabla \times \mathbf{H}(\mathbf{r})+i k_{0} \mathbf{D}(\mathbf{r}), \quad \delta \mathbf{I}_{m}(\mathbf{r})=\nabla \times \mathbf{E}(\mathbf{r})-i k_{0} \mathbf{B}(\mathbf{r}), \quad \mathbf{r} \in \mathbb{C}_{m}
$$

existing in the volume of each lattice cell, as well as spurious surface currents

$$
\delta \mathbf{K}_{l m}(\mathbf{r})=\hat{\mathbf{n}}_{l m} \times\left[\mathbf{H}_{l}(\mathbf{r})-\mathbf{H}_{m}(\mathbf{r})\right], \quad \delta \mathbf{Q}_{l m}(\mathbf{r})=\hat{\mathbf{n}}_{l m} \times\left[\mathbf{E}_{l}(\mathbf{r})-\mathbf{E}_{m}(\mathbf{r})\right]
$$

over all cell-cell and cell-air interfaces. Here $\mathbb{C}_{m}$ is the $m$-th cell and $\hat{\mathbf{n}}_{l m}$ is the unit normal on the interface between the two neighboring cells $\mathbb{C}_{l}$ and $\mathbb{C}_{m}$, pointing from $\mathbb{C}_{l}$ to $\mathbb{C}_{m}$, and subscripts $l$ and $m$ refer to the fields on each side of the boundary. The spurious surface currents $\delta \mathbf{K}_{l m}, \delta \mathbf{Q}_{l m}$ flow on cell-cell or cell-air interfaces $\mathbb{S}_{l m}$. (For uniformity of notation, air regions are also treated as "cells".) In the air, coarse-level fields, by our construction, have the same far-field pattern as the actual fields around the metamaterial sample for a given illumination [8].

It is natural to approximate fine-level fields via a basis set of Bloch waves traveling in different directions: ${ }^{2}$

$$
\mathbf{e}_{m \alpha}(\mathbf{r})=\tilde{\mathbf{e}}_{m \alpha}(\mathbf{r}) \exp \left(i \mathbf{q}_{m \alpha} \cdot \mathbf{r}\right), \mathbf{h}_{m \alpha}=\tilde{\mathbf{h}}_{m \alpha}(\mathbf{r}) \exp \left(i \mathbf{q}_{m \alpha} \cdot \mathbf{r}\right),
$$

where index $\alpha$ labels both the wave vector and the polarization state of the Bloch wave in a lattice cell $m ; \tilde{\mathbf{e}}_{m \alpha}(\mathbf{r})$, $\tilde{\mathbf{h}}_{m \alpha}(\mathbf{r})$ are the respective lattice-periodic factors. As the notation indicates, the basis is defined cell-wise; different bases in different lattice cells could be used. This makes the homogenization problem tractable and reducible to a single cell, rather than global and encompassing the whole sample. An additional benefit of cell-wise bases is that, for a given number of basis functions, local approximations are in general more accurate than global ones (the latter being a particular case of the former when the basis sets are chosen to be the same in all cells).

On the coarse scale, a natural counterpart of the fine-scale Bloch basis is a set of generalized plane waves

$$
\Psi_{m \alpha}=\left\{\mathbf{E}_{m \alpha}, \mathbf{H}_{m \alpha}\right\}=\left\{\mathbf{E}_{0 m \alpha}, \mathbf{H}_{0 m \alpha}\right\} \exp \left(i \mathbf{q}_{m \alpha} \cdot \mathbf{r}\right)
$$

which satisfy Maxwell's equations in a homogeneous but possibly anisotropic medium; subscript ' 0 ' indicates the field amplitudes to be determined. Our objective is to minimize the spurious surface and volume currents (2), (3); the adjustable parameters at our disposal are the wave vectors and the amplitude of the coarse-level waves. This minimization cannot be carried out exactly (other than by brute-force global numerical simulations) because the fields in all lattice cells, as well as the spurious surface and volume currents, are coupled. However, a good approximation can be found via a few natural simplification steps.

First, to avoid unnecessary phase errors, one takes the coarse-level wave vector for each plane wave to be the same as its counterpart for the corresponding Bloch wave, which is already reflected in our notation above (4), (5). Secondly, to minimize the effects of spurious surface currents on the cell-cell and cell-air interfaces, the best that can be done is to take the amplitudes $\left\{\mathbf{E}_{0 m \alpha}, \mathbf{H}_{0 m \alpha}\right\}$ of each plane wave as the boundary average of the tangential components of the respective fine-scale Bloch wave:

$$
\mathbf{E}_{0 m \alpha}=\mathcal{A}_{m}^{\tau} \tilde{\mathbf{e}}_{m \alpha}, \quad \mathbf{H}_{0 m \alpha}=\mathcal{A}_{m}^{\tau} \tilde{\mathbf{h}}_{m \alpha}
$$

The averaging operator $\mathcal{A}_{m}^{\tau}$ for tangential components of a generic vector field $\mathbf{f}$, and a similar operator $\mathcal{A}_{m}^{n}$ for normal components, are defined, in the case of an orthorhombic cell $\mathbb{C}_{m}$, as follows:

$$
\left(\mathcal{A}_{m}^{\tau}\right)_{\gamma} \mathbf{f} \equiv \frac{\int_{\partial \mathbb{C}_{m}} f_{\gamma}\left|\hat{\mathbf{n}} \times \hat{\mathbf{r}}_{\gamma}\right| d S}{\int_{\partial \mathbb{C}_{m}}\left|\hat{\mathbf{n}} \times \hat{\mathbf{r}}_{\gamma}\right| d S}, \quad\left(\mathcal{A}_{m}^{n}\right)_{\gamma} \mathbf{f} \equiv \frac{\int_{\partial \mathbb{C}_{m}} f_{\gamma}\left|\hat{\mathbf{n}} \cdot \hat{\mathbf{r}}_{\gamma}\right| d S}{\int_{\partial \mathbb{C}_{m}}\left|\hat{\mathbf{n}} \cdot \hat{\mathbf{r}}_{\gamma}\right| d S,}, \quad \gamma=1,2,3 ; \quad \hat{\mathbf{r}}_{1,2,3}=\hat{x}, \hat{y}, \hat{z}
$$

\footnotetext{
${ }^{2}$ The basis should in principle also account for surface waves at the material-air interface; this complicated subject [10, 11, 12] remains out of the scope of the paper.
} 
(here $\left|\hat{\mathbf{n}} \times \hat{\mathbf{r}}_{\gamma}\right|$ and $\left|\hat{\mathbf{n}} \cdot \hat{\mathbf{r}}_{\gamma}\right|$ act simply as Kronecker deltas for the faces of the cell parallel or perpendicular to a given coordinate direction $\hat{\mathbf{r}}_{\gamma}, \gamma=1,2,3$.) Note that the averages in (6) involve the periodic factor of the Bloch wave. The amplitudes $\mathbf{E}_{0 m \alpha}, \mathbf{H}_{0 m \alpha}$, along with the Bloch wave vector, define the coarse-level basis function $\alpha$ in a lattice cell $m$.

The next step of our procedure is to minimize the spurious volume currents (2), (3). Omitting the formal mathematical derivation of [8], let us fast forward to the final result - a system of algebraic equations which admits an intuitive interpretation (Fig. 2). Each column of the rightmost rectangular matrix (call it $\Psi_{E H}$ ) corresponds to a given coarse-level basis function (mode) $\alpha$, and the entries of that column are the $x y z$-components of the wave amplitudes $\mathbf{E}_{0 m \alpha}, \mathbf{H}_{0 m \alpha}$. The number of columns $n$ is equal to the chosen number of basis functions. As a practical guide, for a generic 3D problem one could take $n=12$ (waves traveling in the $\pm x, \pm y, \pm z$ directions, with two possible polarizations per direction); but bigger bases are also possible, with some trade-offs discussed below. Obviously, in special cases when some of the field components are zero (e.g. $s$ or $p$ modes), dimensions of $\Psi_{E H}$ can be reduced accordingly. The leftmost rectangular matrix (call it $\Psi_{D B}$ ) is completely analogous to $\Psi_{E H}$ and contains the DB amplitudes derived from Maxwell's curl equations, viz.:

$$
\mathbf{B}_{0 m \alpha}=k_{0}^{-1} \mathbf{q}_{m \alpha} \times \mathbf{E}_{0 m \alpha}, \quad \mathbf{D}_{0 m \alpha}=-k_{0}^{-1} \mathbf{q}_{m \alpha} \times \mathbf{H}_{0 m \alpha}
$$

The (local) material tensor is represented by a $6 \times 6$ matrix. Since the number of columns in matrix $\Psi_{E H}$ is typically greater than the number of rows, the matrix equation for the material tensor is solved in the least squares sense:

$$
\mathcal{M}=\Psi_{D B} \Psi_{E H}^{+} ; \quad \delta_{\text {l.s. }}=\left\|\Psi_{D B}-\mathcal{M} \Psi_{E H}\right\|
$$

where $\Psi_{E H}^{+}$is the Moore-Penrose pseudoinverse of $\Psi_{E H}, \delta_{\text {l.s. }}$ is the associated least-squares error, and $\|\cdot\|$ is a suitable matrix norm whose choice is not of critical importance.

If more functions are included in the basis, then finescale fields can, generally speaking, be approximated more accurately; but, as a trade-off, matrices $\Psi_{D B}$ and $\Psi_{E H}$ become "more rectangular," leading in general to a higher leastsquares error $\delta_{\text {l.s. }}$. A high error indicates the existence of many physically different modes not simultaneously and accurately representable by the same material tensor. This is not a deficiency of the proposed methodology but rather a principal limitation of local homogenization due to the complexity of fields in a lattice cell.

An alternative intuitive interpretation of (9) and Fig. 2 is in terms of "information compression". Namely, (partial) information about fields in the lattice cell is contained in the chosen set of $n$ modes. If these modes are significantly different and $n \gg 6$, this information cannot be compressed without loss to a $6 \times 6$ matrix.

From these qualitative observations, one can see two ways

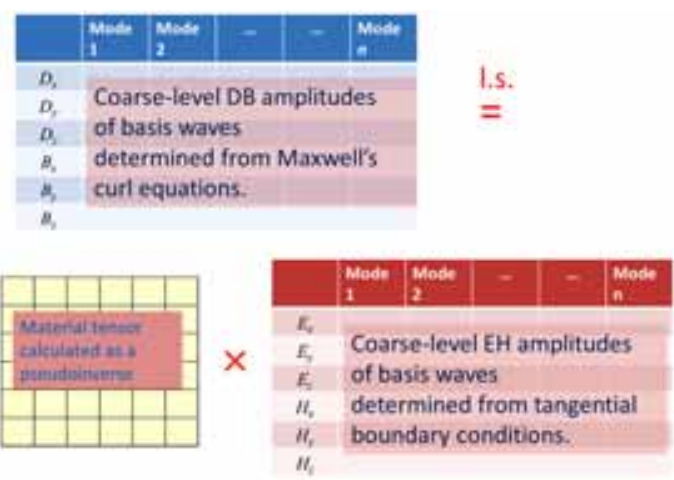

Figure 2. A schematic representation of the matrix equation $\Psi_{D B} \stackrel{\text { l.s. }}{=} \mathcal{M} \Psi_{E H}$. '1.s.' stands for 'lest squares'. of reducing the error (9) - that is, ways of improving the accuracy of homogenization. First, one can reduce the number of columns in $\Psi_{D B}$ and $\Psi_{E H}$, thereby limiting the set of basis modes. The material tensor will then be tailored to a subset of illumination conditions representable by those modes. An extreme example is consideration of normal incidence only, in which case the matrices will have only four columns (two opposite directions of propagation and two polarizations), and infinitely many material tensors will render $\delta_{\text {l.s. }}=0$.

A complementary possibility of reducing the error is to expand the number of rows in the matrices. This amounts to considering, in addition to field amplitudes, other degrees of freedom (dof) - e.g. integral ones. This way of making $\Psi_{E H}$ "more square" leads to nonlocal homogenization (Sections 3 and 5).

\section{Nonlocal Homogenization}

A nonlocal material relationship between two fields is typically written in the form

$$
\mathbf{D}(\mathbf{r})=\int_{\Omega} \mathcal{E}\left(\mathbf{r}, \mathbf{r}^{\prime}\right) \mathbf{E}\left(\mathbf{r}^{\prime}\right) d \Omega
$$


where $\mathcal{E}$ is a convolution kernel and $\Omega$ is the region occupied by the material in question. A local relationship is an obvious particular case if the kernel is the Dirac delta. In the physical literature, nonlocal models are typically derived and used in Fourier space (k-space); this is common, in particular, in plasma physics [13], crystal optics [14], and plasmonics. However, the use of Fourier transforms relies on translational invariance of the convolution kernel, which does not hold in the presence of interface boundaries. Yet interface conditions must be accounted for, as they determine the effective impedance and hence magnetic effects $[8,15,16]$. One can use V. A. Markel's idea [17] to restrict the dependence of the integral kernel in the vicinity of a boundary to the tangential directions $\tau$ only; $\mathcal{E}\left(\mathbf{r}, \mathbf{r}^{\prime}\right)=\mathcal{E}\left(\hat{\mathbf{n}} \times \mathbf{r}, \hat{\mathbf{n}} \times \mathbf{r}^{\prime}\right)$. A natural choice is a Gaussian kernel $\mathcal{E}\left(\mathbf{r}, \mathbf{r}^{\prime}\right)=\mathcal{E}_{0} \exp \left(-\left|\hat{\mathbf{n}} \times\left(\mathbf{r}-\mathbf{r}^{\prime}\right)\right|^{2} / \tau_{0}^{2}\right)$, where the amplitude $\mathcal{E}_{0}$ and width $\tau_{0}$ are adjustable parameters, and $\hat{\mathbf{n}}$ is the unit normal vector.

A schematic representation of the nonlocal model in matrix form is shown in Fig. 3. The key amendment to the local model (Fig. 2) is additional degrees of freedom leading to the corresponding expansion of $\Psi_{E H}$ downward and of the material tensor rightward. The tensor thus acquires, in addition to its local part, a nonlocal one. This is illustrated with an example in Section 5.

\section{The Clausius-Mossotti Model Revisited}

Although in the previous sections our homogenization framework was described in the context of full Maxwell's electrodynamics, the concepts are applicable in electrostatics as well, albeit with some changes. In particular, since the Bloch "wavevector" in the static case is zero, the simple choice of Bloch basis functions reduces just to two (2D) or three (3D) polarization states. This is sufficient for a basic theory; further enhancements are certainly possible.

The ideas outlined in this paper are very different, at least at first glance, from those of classical effective medium theories. We have made no mention of e.g. dipole moments, polarization, Lorentz local fields, volume averages, and so on. It is therefore instructive to see how classical theories fit the general methodology outlined above.

As a particular but interesting case, consider a very large
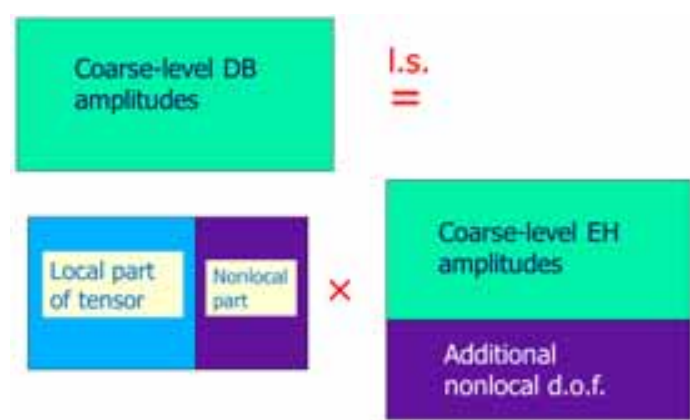

Figure 3. A schematic representation of the matrix equation in the nonlocal case. The equation is analogous to that of Fig. 2, but the matrices are expanded, as described in the text. square lattice of identical point dipoles, each of which has a polarizability $\alpha$; that is, its dipole moment is $\mathbf{p}=\alpha \mathbf{E}^{\prime}$, where $\mathbf{E}^{\prime}$ is the field at the location of the dipole, due to all other sources. The $3 \mathrm{D}$ case is completely analogous. Let the dipole array be immersed in a uniform external ("incident") field $\mathbf{E}_{\text {inc }}$ in the $x$ direction, and let us focus on a lattice cell $\left[-\frac{1}{2} a, \frac{1}{2} a\right]^{3}$ in the middle of the array ("in the bulk"); $a$ is the cell size, and the origin for convenience is placed at the center of the cell. It is easy to see that each of the four faces of the cell parallel to the $x$ axis (i.e. to the direction of the applied field) lies in a symmetry plane of the structure and of the electrostatic potential. ${ }^{3}$ Similarly, the two faces perpendicular to $x$ lie in antisymmetry planes. It then follows that the potential within a cell "in the bulk" is subject to the following boundary conditions (see e.g. [18] for a mathematical presentation):

$\hat{\mathbf{n}} \cdot \mathbf{d}=0$, Neumann; face parallel to applied field; $\hat{\mathbf{n}} \times \mathbf{e}=0$, Dirichlet; face perpendicular to applied field

where $\hat{\mathbf{n}}$ is the unit normal vector on the cell boundary (undefined at the edges and corners). The differential equation for the potential $u$ within the cell is

$$
\nabla^{2} u=-4 \pi \rho, \quad \rho=-\partial_{x} \delta(\mathbf{r})
$$

where $\delta(\mathbf{r})$ is the Dirac delta-function residing at the center of the cell and $-\partial_{x} \delta$ is a unit dipole. The boundary value problem with sources (12) and conditions (11) defines the potential and field in the cell uniquely, up to a multiplicative constant. This potential can be taken as one of the basis functions, $\psi_{1}(\mathbf{r})$, on the fine scale. The other basis function, $\psi_{2}(\mathbf{r})$, is defined in a similar way, for the applied field in the $y$ direction.

\footnotetext{
${ }^{3}$ This is strictly true for an infinite array and approximately true for a very large array.
} 
At the coarse level, the corresponding functions, by construction, satisfy the electrostatic equations in a homogeneous medium and can be taken to be simply constant and, as before, are found as the boundary averages of the respective fine-level fields.

The electrostatic problem with the boundary conditions (11) is well known, and various solutions have been put forward in the literature. Once this solution has been found one way or another, (9) assumes the form

$$
\left(\begin{array}{ll}
\mathcal{E}_{x x} & \mathcal{E}_{x y} \\
\mathcal{E}_{y x} & \mathcal{E}_{y y}
\end{array}\right)\left(\begin{array}{ll}
\left(\mathcal{A}^{\tau}\right)_{x} \mathbf{e}_{1} & \left(\mathcal{A}^{\tau}\right)_{x} \mathbf{e}_{2} \\
\left(\mathcal{A}^{\tau}\right)_{y} \mathbf{e}_{1} & \left(\mathcal{A}^{\tau}\right)_{y} \mathbf{e}_{2}
\end{array}\right)=\left(\begin{array}{ll}
\left(\mathcal{A}^{n}\right)_{x} \mathbf{d}_{1} & \left(\mathcal{A}^{n}\right)_{x} \mathbf{d}_{2} \\
\left(\mathcal{A}^{n}\right)_{y} \mathbf{d}_{1} & \left(\mathcal{A}^{n}\right)_{y} \mathbf{d}_{2}
\end{array}\right)
$$

The $\mathcal{E}$ tensor can be found from this equation directly by matrix inversion. Note that in this particular example all matrices in (13) are actually diagonal due to geometric symmetry, so the inversion is trivial.

Next, we argue that the Clausius-Mossotti (C-M) theory can be interpreted as a way of approximating the circulations and fluxes of the basis functions $\psi$, as required in (13). The key quantities in C-M, as shown e.g. in [19], are (i) polarization and (ii) the volume integral of the electric field within the cell. Although the relation between these quantities and the ones in (13) may not be immediately obvious, it is in fact straightforward [20]. Indeed, consider any rectangular loop with two edges lying on the opposite "Dirichlet" faces of the cell; these edges (call them edges $1,2)$ are perpendicular to the applied field. The other two edges of the loop (edges 3,4) cut through the cell and can be located anywhere in the volume of the cell; they are parallel to the applied field. Since the circulation of the field over edges 1,2 is zero due to the Dirichlet condition, it is clear that the line integrals of the field over edges 3, 4 (taken in the same direction) are equal. From that, it is easy to deduce that the volume average of the electric field over the cell is equal to the boundary average of its tangential component - i.e. exactly the quantity needed in (13). In other words, the volume average in this case is a perfect proxy for the boundary average. The calculation of the volume integral must account for the singular term in the dipole field [19] and yields in the 2D case under consideration

$$
\mathcal{A}^{\tau} \mathbf{e}_{1}=\left\langle\mathbf{e}_{1}\right\rangle_{\mathbb{C}}=\mathbf{E}_{\text {inc }}-2 \pi \mathbf{P}_{1}, \quad \mathbf{P}_{1} \equiv\left\langle\mathbf{p}_{1}(\mathbf{r})\right\rangle_{\mathbb{C}}
$$

A similar relation of course exists for the second basis function, i.e. between $\mathbf{e}_{2}$ and $\mathbf{P}_{2}$. The derivation also relies on the fact that the volume average of the field produced by all dipoles outside the cell is zero. This is true for an infinite lattice due to symmetry and approximately true for a very large one.

Now let us turn to the normal component of $\mathbf{D}$ and flux through the boundary. Consider the volume contained between two arbitrary cross-sections of the cell perpendicular to the applied field (in our case, perpendicular to the $x$ axis). Because of the Neumann boundary conditions on the four faces of this volume, it is clear that the flux through the two cross-sections must be the same. Hence the flux of $\mathbf{D}$ through any cross-section perpendicular to $x$ is the same, and face averages in (13) can be replaced with volume averages, and we have

$$
\mathcal{A}^{n} \mathbf{d}(\mathbf{r})=\langle\mathbf{d}(\mathbf{r})\rangle_{\mathbb{C}}=\langle\mathbf{e}(\mathbf{r})+4 \pi \mathbf{p}(\mathbf{r})\rangle_{\mathbb{C}}=\mathbf{E}_{0}-2 \pi \mathbf{P}+4 \pi \mathbf{P}=\mathbf{E}_{0}+2 \pi \mathbf{P}
$$

The Maxwell Garnett formula then immediately follows from (14), (15) and the definition (13) of $\epsilon_{\mathrm{eff}}$ :

$$
\epsilon_{\mathrm{eff}}=\frac{\langle D\rangle_{\mathbb{C}}}{\langle E\rangle_{\mathbb{C}}}=\frac{1+2 \pi \tilde{\alpha}}{1-2 \pi \tilde{\alpha}}, \quad \tilde{\alpha} \equiv \frac{\alpha}{V_{\mathbb{C}}}
$$

\section{Example of the General Methodology: Nonlocal Model of Layered Media}

As an example of nonlocal homogenization, we consider a layered medium periodic in the normal direction $n$ and homogeneous in the plane perpendicular to $n$. Maxwell's equations for $s$-polarization (one-component electric field $e=e_{z}, d=d_{z}$, two-component magnetic field $\left.\mathbf{h}=\hat{n} h_{n}+\hat{\tau} h_{\tau}\right)$ are

$$
\partial_{n} e=-i k_{0} b_{\tau} ; \quad \partial_{\tau} e=i k_{0} b_{n} ; \quad \partial_{n} h_{\tau}-\partial_{\tau} h_{n}=-i k_{0} d
$$

(Analysis and results for $p$-polarization are qualitatively similar.) Let the constitutive relation in a homogenized nonlocal medium be

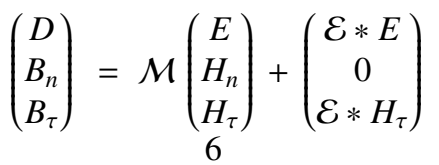



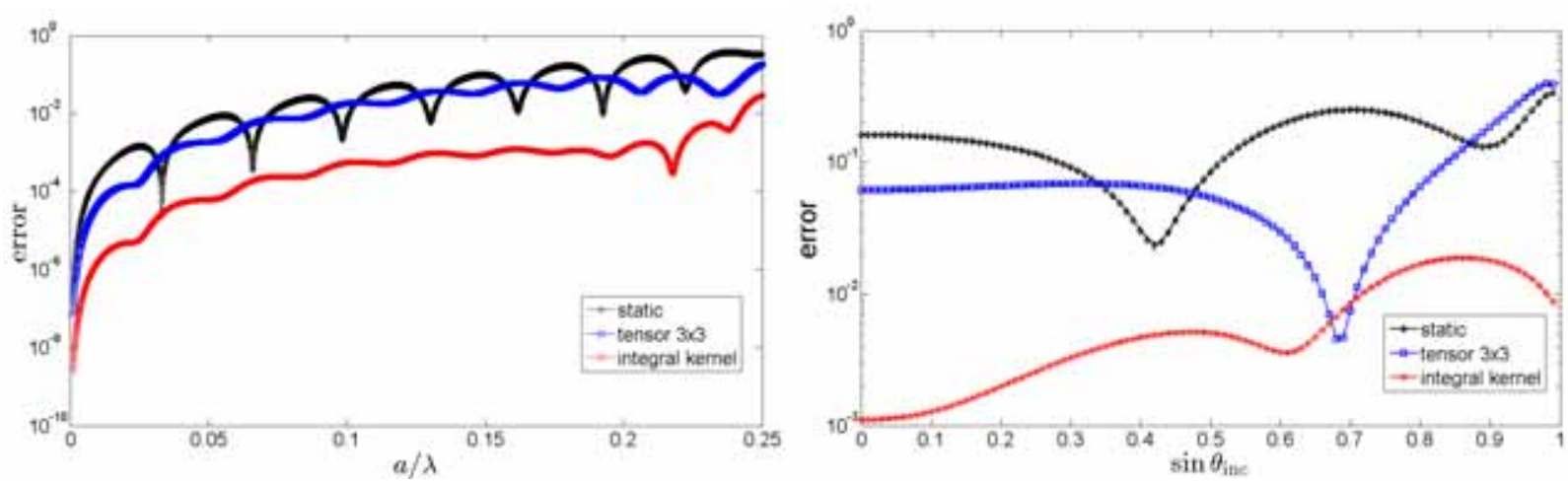

Figure 4. Example A of a layered medium from [16, 8]. Absolute error in $R$ vs. free-space wavelength (left) and vs. the sine of the angle of incidence (right); nonlocal homogenization. The lattice cell contains three layers of widths $a / 4, a / 2$ and $a / 4$, with scalar permittivities $\epsilon_{1}$, $\epsilon_{2}$, and $\epsilon_{1}$, respectively. $\left(\epsilon_{1}=4+0.1 i\right.$ and $\epsilon_{2}=1$.) Fine-level basis: $2 n_{\mathrm{dir}}$ Bloch modes traveling at $n_{\mathrm{dir}}=7 \mathrm{different}$ angles in $(-\pi / 2, \pi / 2) ; n_{\mathrm{dir}}=7$. The kernel width parameter $\tau_{0}=a$.

where ' $*$ ' denotes convolution with some integral kernel $\mathcal{E}=\mathcal{E}(\tau)$ depending only on the tangential coordinate; e.g. $(\mathcal{E} * E)(\tau, n)=\int_{\tau^{\prime}=-\infty}^{\infty} E\left(\tau^{\prime}, n\right) \mathcal{E}\left(\tau-\tau^{\prime}\right) d \tau^{\prime}$. For simplicity, in this model the kernel is scalar (non-tensorial), and the nonlocality is confined to the tangential field components only. We seek coarse-level fields as a generalized plane wave $E=E_{0} \exp (i \mathbf{q} \cdot \mathbf{r})$, etc. Simple algebra reduces the above equations to a linear eigenproblem with respect to $\tilde{q}_{n}$ for a fixed $\tilde{q}_{\tau}$ :

$$
\begin{gathered}
\tilde{q}_{n} Q_{n} \Psi=\left[P\left(\mathcal{M}+\tilde{\mathcal{E}}\left(\tilde{q}_{\tau}\right) I_{101}\right)-\tilde{q}_{\tau} Q_{\tau}\right] \Psi \\
P=\left(\begin{array}{ccc}
0 & 0 & -1 \\
0 & 1 & 0 \\
1 & 0 & 0
\end{array}\right), \quad Q_{n}=\left(\begin{array}{ccc}
1 & 0 & 0 \\
0 & 0 & 0 \\
0 & 0 & -1
\end{array}\right), \quad Q_{\tau}=\left(\begin{array}{lll}
0 & 0 & 0 \\
1 & 0 & 0 \\
0 & 1 & 0
\end{array}\right), \quad \Psi=\left(\begin{array}{c}
E \\
H_{n} \\
H_{\tau}
\end{array}\right), \quad I_{101}=\left(\begin{array}{ccc}
1 & 0 & 0 \\
0 & 0 & 0 \\
0 & 0 & 1
\end{array}\right), \quad \tilde{q}_{n, \tau}=k_{0}^{-1} q_{n, \tau}
\end{gathered}
$$

Recall, in particular, that for a Gaussian kernel $\mathcal{E}(\tau)=\pi^{-1 / 2} \tau_{0}^{-1} \exp \left(-\tau^{2} / \tau_{0}^{2}\right)$ - with a given width parameter $\tau_{0}$ and area normalized to unity - the Fourier transform is $\tilde{\mathcal{E}}(k)=\exp \left(-k^{2} \tau_{0}^{2} / 4\right)$.

Integral-kernel nonlocal homogenization outlined in the previous section was performed, with the additional two dof of the form $\int_{\mathbb{R}} W\left(\tau^{\prime}\right) \mathcal{E}\left(\tau^{\prime}\right) d \tau^{\prime}$, where $W$ is either $E$ or $H_{\tau}$. The respective nonlocal tensor can be represented as a $3 \times 6$ matrix (see Fig. 3 as a visual aid).

In $[8,16]$, we introduced several benchmark examples of layered media and explored their non-asymptotic but local homogenization. This section extends this to nonlocal homogenization using Example A of these papers. The medium consists of a finite number of stacked inversion-symmetric lattice cells each of which contains three intrinsically nonmagnetic layers of widths $a / 4, a / 2$ and $a / 4$ and scalar permittivities $\epsilon_{1}, \epsilon_{2}$, and $\epsilon_{1}$, respectively $\left(\epsilon_{1}=4+0.1 i\right.$ and $\left.\epsilon_{2}=1\right)$. We assume that $\lambda$ is fixed and $a$ changes. The numerical results depend only weakly on the kernel width parameter $\tau_{0}$ when it varies within a reasonable range from $\sim 10-20 \%$ of the cell size $a$ to several cell sizes. To avoid any artificial fine-tuning of this parameter, it was simply set to $\tau_{0}=a$.

Eigenproblem (17) could be solved for $\tilde{q}_{n}$ and the eigenvector $\Psi$ for a given tensor $\mathcal{M}$ and $\tilde{q}_{\tau}$ analytically, but in practice it is more convenient to solve it numerically and obtain two solutions (backward and forward waves) in the slab. These waves are then coupled in the usual way with the incident, reflected and transmitted waves outside the slab, yielding the reflection and transmission coefficients. ${ }^{4}$

The fine-scale basis consisted of $2 n_{\text {dir }}$ Bloch modes traveling at $n_{\text {dir }}=7$ different angles in $(-\pi / 2, \pi / 2)$ (two Bloch waves - "back" and "forth" - per angle). The coarse-level EH-amplitudes are the boundary averages (6) of the corresponding eh-fields; the DB magnitudes are found from Maxwell's equations; see (8).

\footnotetext{
${ }^{4}$ For more general 2D and 3D problems, Maxwell's equations in conjunction with nonlocal constitutive relations similar to (16) can be solved using traditional numerical methods such as finite element or finite difference analysis. However, less traditional Trefftz approximations, where a set of eigenmodes of (17) form a basis, can be much more efficient (see [21] and references there).
} 
Fig. 4 shows the absolute errors in the reflection coefficient: in the left panel - as a function of $a / \lambda$ in the first photonic band, and in the right panel - as a function of $\sin \theta_{\text {inc }}$ for $a / \lambda=0.2$, for a metamaterial slab with a 10-cell thickness in the normal direction. It can be seen that the errors of the nonlocal homogenization are far lower than those of local homogenization and of the standard static (asymptotic) homogenization.

It should be noted that the relative simplicity of layered media is deceiving; their homogenization is notoriously difficult. A number of papers on this subject have been published ([22, 23, 24] and more); yet I am not aware of any method with a level of accuracy for transmission and reflection comparable with that of Fig. 4; the published results improve the accuracy only of the dispersion relation at best.

Some ideas of our non-asymptotic and nonlocal homogenization accord nicely with those of other publications, but there are nevertheless substantial differences. For example, the use of a Bloch wave basis to generalize impedance boundary conditions is key in [25, 26], but not in the context of homogenization. On the other hand, "high frequency homogenization" of [27] represents the dispersion relations beyond their asymptotic limit but does not deal with boundary impedances.

\section{Conclusion}

Future research in metamaterials will undoubtedly involve development of advanced homogenization theories. The paper outlines a promising path in that direction - a methodology valid in both electrostatics and electrodynamics and applicable to an arbitrary size and composition of the lattice cell. Classical theories (Clausius-Mossotti, LorenzLorentz, Maxwell Garnett) fit well into the proposed new framework and are used to illustrate the concept. Nonlocal effects can be included in the model, making an order-of-magnitude accuracy improvements possible. One may note a hierarchy of models, with various trade-offs between accuracy and simplicity: (i) static (i.e. asymptotic, $a / \lambda \rightarrow 0$ ) - does not predict nontrivial magnetic effects; (ii) non-asymptotic but local; (iii) nonlocal; (iv) and, finally, full numerical simulations of the whole fine-scale structure of the metamaterial.

A particularly challenging problem for future research is to determine what effective material tensors are attainable for given constituents of a metamaterial with their given properties, and how the lattice cell could be designed to produce such tensors. For example, what is the maximum effective permeability achievable or what is strongest (by some measure) chirality? Bounds for effective parameters are currently known only for relatively simple settings, such as static dielectric permittivity of mixtures with two ingredients. The methodology of this paper may help to make progress toward solving a much broader set of problems of this kind.

This research was supported in part by the US National Science Foundation Grants DMS-1216927 and DMS-1620112.

\section{References}

[1] M. V. Kostin, V. V. Shevchenko, Artificial magnetic material based on ring currents, Sov. J. of Communications Technology and Electronics 33 (12) (1988) 38-42.

[2] I. Tsukerman, Negative refraction and the minimum lattice cell size, J. Opt. Soc. Am. B 25 (2008) $927-936$.

[3] A. Bossavit, G. Griso, B. Miara, Modelling of periodic electromagnetic structures bianisotropic materials with memory effects, J. Math. Pures \& Appl. 84 (7) (2005) 819-850.

[4] D. Sjöberg, C. Engstrom, G. Kristensson, D. J. N. Wall, N. Wellander, A Floquet-Bloch Decomposition of Maxwell's Equations Applied to Homogenization, Multiscale Modeling \& Simulation 4 (1) (2005) 149-171. doi:10.1137/040607034. URL http://link . aip.org/link/?MMS/4/149/1

[5] L. Lewin, The electrical constants of a material loaded with spherical particles, Proc. Inst. Elec. Eng. 94 (1947) 65-68.

[6] N. A. Khizhnyak, Artificial anisotropic dielectrics formed from two-dimensional lattices of infinite bars and rods, Sov. Phys. Tech. Phys. 29 (1959) 604-614.

[7] P. C. Waterman, N. E. Pedersen, Electromagnetic scattering by periodic arrays of particles, J. Appl. Phys. 59 (1986) $2609-2618$.

[8] I. Tsukerman, V. A. Markel, A nonasymptotic homogenization theory for periodic electromagnetic structures, Proc Royal Society A 470 (2014) 2014.0245. doi : 10.1098/rspa.2014.0245.

[9] P. Penfield, H. A. Haus, Electrodynamics of Moving Media, The MIT Press, 1967.

[10] X. Y. Xiong, L. J. Jiang, V. A. Markel, I. Tsukerman, Surface waves in three-dimensional electromagnetic composites and their effect on homogenization, Optics Express 21 (9) (2013) 10412-10421.

[11] V. A. Markel, J. C. Schotland, Homogenization of Maxwell's equations in periodic composites: Boundary effects and dispersion relations, Phys. Rev. E 85 (2012) 066603. doi:10.1103/PhysRevE.85.066603. URL http://link.aps.org/doi/10.1103/PhysRevE.85.066603

[12] X. Xiong, L. L. Meng, L. J. Jiang, W. Sha, F. Yang, Efficient calculation of large finite periodic structures based on surface wave analysis, IEEE Transactions on Antennas and Propagation 63 (1) (2015) 69-80. doi:10.1109/TAP. 2014.2365211. 
[13] V. L. Ginzburg, Propagation of electromagnetic waves in plasma, Gordon and Breach, 1962

[14] V. M. Agranovich, V. L. Ginzburg, Crystal Optics with Spatial Dispersion, and Excitons, Berlin; New York: Springer-Verlag, 1984.

[15] I. Tsukerman, Effective parameters of metamaterials: a rigorous homogenization theory via Whitney interpolation, J Opt Soc Am B 28 (3) (2011) 577-586.

[16] V. A. Markel, I. Tsukerman, Current-driven homogenization and effective medium parameters for finite samples, Phys. Rev. B 88 (2013) 125131. doi:10.1103/PhysRevB.88.125131.

URL http://link.aps.org/doi/10.1103/PhysRevB.88.125131

[17] V. A. Markel, Private communication (2016).

[18] N. S. Bakhvalov, G. Panasenko, Homogenisation: Averaging Processes in Periodic Media, Mathematical Problems in the Mechanics of Composite Materials, Springer, 1989.

[19] V. A. Markel, Introduction to the Maxwell Garnett approximation: tutorial, J. Opt. Soc. Am. A 33 (7) (2016) 1244-1256. doi:10.1364/ JOSAA. 33.001244.

URL http: //josaa.osa. org/abstract. cfm?URI=josaa-33-7-1244

[20] D. J. Bergman, The dielectric constant of a composite material - a problem in classical physics, Physics Reports 4 (9) (1978) $377-407$.

[21] F. Kretzschmar, S. M. Schnepp, H. Egger, F. Ahmadi, N. Nowak, V. Markel, I. Tsukerman, The Power of Trefftz Approximations: Finite Difference, Boundary Difference and Discontinuous Galerkin Methods; Nonreflecting Conditions and Non-Asymptotic Homogenization, Vol. 9045, Springer Series: Lecture Notes in Computer Science, 2015, pp. 47-58.

[22] A. V. Chebykin, A. A. Orlov, C. R. Simovski, Y. S. Kivshar, P. A. Belov, Nonlocal effective parameters of multilayered metal-dielectric metamaterials, Phys. Rev. B 86 (2012) 115420. doi:10.1103/PhysRevB.86.115420. URL http://link.aps.org/doi/10.1103/PhysRevB.86.115420

[23] R.-L. Chern, Spatial dispersion and nonlocal effective permittivity for periodic layered metamaterials, Opt. Express 21 (14) (2013) 1651416527. doi:10.1364/0E.21.016514.

URL http: //www . opticsexpress . org/abstract . cfm?URI=oe-21-14-16514

[24] L. Sun, Z. Li, T. S. Luk, X. Yang, J. Gao, Nonlocal effective medium analysis in symmetric metal-dielectric multilayer metamaterials, Phys. Rev. B 91 (2015) 195147. doi:10.1103/PhysRevB.91.195147. URL http://link.aps.org/doi/10.1103/PhysRevB.91.195147

[25] F. J. Lawrence, L. C. Botten, K. B. Dossou, C. M. de Sterke, Antireflection coatings for two-dimensional photonic crystals using a rigorous impedance definition, Applied Physics Letters 93 (12) (2008) 121114. arXiv:http://dx.doi.org/10.1063/1.2992066, doi:10. 1063/1.2992066

URL http://dx.doi.org/10.1063/1.2992066

[26] T. Antonakakis, R. V. Craster, S. Guenneau, High-frequency homogenization of zero-frequency stop band photonic and phononic crystals, New Journal of Physics 15 (10) (2013) 103014. URL http: //stacks.iop.org/1367-2630/15/i=10/a=103014

[27] R. Craster, J. Kaplunov, E. Nolde, S. Guenneau, Bloch dispersion and high frequency homogenization for separable doubly-periodic structures, Wave Motion 49 (2) (2012) 333 - 346. doi : http: //dx . doi .org/10.1016/j . wavemoti.2011.11.005. URL http://www.sciencedirect.com/science/article/pii/S0165212511001417 